April 2002

\title{
(Anti-) de Sitter Black Holes in higher derivative gravity and dual Conformal Field Theories
}

\author{
SHIN'ICHI NOJIRI and SERGEI D. ODINTSOV^] \\ Department of Applied Physics \\ National Defence Academy, Hashirimizu Yokosuka 239, JAPAN \\ A Tomsk Pedagogical University, 634041 Tomsk, RUSSIA
}

\begin{abstract}
Thermodynamics of five-dimensional Schwarzschild Anti-de Sitter (SAdS) and SdS black holes in the particular model of higher derivative gravity is considered. The free energy, mass (thermodynamical energy) and entropy are evaluated. There exists the parameters region where $\mathrm{BH}$ entropy is zero or negative. The arguments are given that corresponding $\mathrm{BH}$ solutions are not stable. We also present the FRW-equations of motion of time (space)like branes in SAdS or SdS BH background. The properties of dual CFT are discussed and it is shown that it has zero Casimir energy when BH entropy (effective gravitational constant) is zero. The Cardy-Verlinde formula for CFT dual to SAdS or SdS BH is found in the universal form.
\end{abstract}

\footnotetext{
${ }^{1}$ nojiri@cc.nda.ac.jp

2 odintsov@mail.tomsknet.ru
} 


\section{Introduction}

AdS/CFT correspondence [1] and proposed dS/CFT correspondence [2, 3, [4](for recent study see refs. [5, 6, 6, 8] and references therein) provided quite deep motivation to study AdS and dS black hole thermodynamical properties, respectively. The additional related motivation comes from the brane-world approach. It is quite well-known that five-dimensional AdS Einstein gravity gives the description of dual four-dimensional CFT in the leading order of large $N$ approximation. Higher derivative gravity is natural framework to investigate the non-perturbative properties of dual CFT in next-to-leading order. The study of AdS and dS black holes in five-dimensional classical higher derivative gravity [9] demonstrated the possibility of negative entropy black holes [8]. This is quite unusual situation which is not typical in Einstein gravity. It is caused mainly by consideration of the higher derivative terms coefficients on the equal footing with gravitational constant.

From the thermodynamical point of view the objects with negative entropy are not physical ones and they should not occur. Hence, it is expected that there should be some mechanism to suppress the appearence of such objects. Currently, one can provide the following points of view to resolve this problem.

1. Superstring/M-theory compactifications should predict the physical values of the higher derivative terms coefficients in the gravitational action. Presumably, the corresponding values are such that region of parameters admitting negative entropy black holes is never realized in physical world. This point of view was suggested in ref. [8]. However, so far there is no explicit realization of realistic compactified theory.

2. Quantum higher derivative gravity which is renormalizable in four dimensions (for review, see [10]) is famous due to non-unitarity problem. This may indicate that higher derivative terms should be accounted only at microscopic QG scale. In other words, strange classical effects of higher derivative gravity should be suppressed quantum mechanically.

3. The negative entropy black holes may indicate to the breaking of some physical picture. For example, the appearence of such objects may indicate to the possibility of some new sort of phase transition (say, between de Sitter and Anti-de Sitter black holes [8]). If so, some manifestation of such phase transition should be seen in dual CFT description. Moreover, negative entropy black hole solutions should not be stable. Finally, the resolution may 
lie in the combination of above points.

The purpose of this work is to study the thermodynamics (entropy, free energy, mass) of five-dimensional AdS and dS black holes in sufficiently simple model of higher derivative gravity. Nevertheless, such model admits the realization of negative entropy black holes. We explicitly show that negative entropy black holes are not stable. The FRW equations of motion of time (space)-like branes in SAdS or SdS black holes are considered. The study of dual CFT shows that when BH entropy is zero, the brane CFT energy is also zero. The Cardy-Verlinde formula for (A)dS BHs is presented..

\section{2 (Anti)-de Sitter Black Hole thermodynam- ics}

In attempt to understand the thermodynamics of $(\mathrm{A}) \mathrm{dS}$ BHs let us first present the short review of such objects in Einstein gravity. The 5dimensional Einstein gravity action is given by,

$$
S=\int d^{5} x \sqrt{-g}\left\{\frac{1}{\kappa^{2}} R-\Lambda\right\} .
$$

When $\Lambda$ is negative, the Schwarzschild anti-de Sitter (SAdS) spacetime is a solution of the Einstein equation:

$$
d s_{\text {Einstein }}^{2}=-\mathrm{e}^{2 \rho} d t^{2}+\mathrm{e}^{-2 \rho} d r^{2}+r^{2} g_{i j} d x^{i} d x^{j}, \quad \mathrm{e}^{2 \rho}=\frac{k}{2}-\frac{\mu_{\mathrm{AdS}}}{r^{2}}+\frac{r^{2}}{l_{\mathrm{AdS}}^{2}} .
$$

Here $g_{i j}$ is the metric of the 3-dimensional Einstein manifold, where the Ricci curvature $R_{i j}$ satisfies the relation $R_{i j}=k g_{i j}$. The case of $k=2$ corresponds to the unit sphere, $k=-2$ to the unit hyperboloid and, as a special case, $k=0$ to the flat space. The length parameter $l_{\text {AdS }}$ is

$$
l_{\text {AdS }}^{2}=-\frac{\kappa^{2} \Lambda}{12} .
$$

On the other hand, when $\Lambda$ is positive, the Schwarzschild- de Sitter (SdS) spacetime is a solution:

$$
d s_{\text {Einstein }}^{2}=-\mathrm{e}^{2 \rho} d t^{2}+\mathrm{e}^{-2 \rho} d r^{2}+r^{2} d \Omega_{3}^{2}, \quad \mathrm{e}^{2 \rho}=1-\frac{\mu_{\mathrm{dS}}}{r^{2}}-\frac{r^{2}}{l_{\mathrm{dS}}^{2}} .
$$


Here $d \Omega_{3}^{2}$ is the metric of the unit 3-dimensional sphere. The length parameter is given by

$$
l_{\mathrm{dS}}^{2}=-l_{\mathrm{AdS}}^{2}=\frac{\kappa^{2} \Lambda}{12} .
$$

There is a black hole horizon at

$$
r^{2}=r_{\mathrm{AdS}-\mathrm{bh}}^{2} \equiv \frac{-\frac{k}{2} l_{\mathrm{AdS}}^{2}+\sqrt{l_{\mathrm{AdS}}^{4}\left(\frac{k}{2}\right)^{2}+4 \mu_{\mathrm{AdS}} l_{\mathrm{AdS}}^{2}}}{2}
$$

for the SAdS spacetime and

$$
r^{2}=r_{\mathrm{dS}-\mathrm{bh}}^{2} \equiv \frac{l_{\mathrm{dS}}^{2}-\sqrt{l_{\mathrm{dS}}^{4}-4 \mu_{\mathrm{dS}} l_{\mathrm{dS}}^{2}}}{2}
$$

for the SdS spacetime. For the Schwarzschild-dS spacetime there is also cosmological horizon at

$$
r^{2}=r_{\mathrm{csm}}^{2} \equiv \frac{l_{\mathrm{dS}}^{2}+\sqrt{l_{\mathrm{dS}}^{4}-4 \mu_{\mathrm{dS}} l_{\mathrm{dS}}^{2}}}{2} .
$$

Then the corresponding Hawking temperatures are given by

$$
T_{\mathrm{AdS}-\mathrm{bh}}=\frac{k}{4 \pi r_{\mathrm{AdS}-\mathrm{bh}}}+\frac{r_{\mathrm{AdS}-\mathrm{BH}}}{\pi l_{\mathrm{AdS}}^{2}}
$$

for the SAdS spacetime and

$$
T_{\mathrm{dS}-\mathrm{bh}, \mathrm{csm}}=\left|\frac{\mu_{\mathrm{dS}}}{2 \pi r_{\mathrm{dS}-\mathrm{bh}, \mathrm{csm}}^{3}}-\frac{r_{\mathrm{dS}-\mathrm{bh}, \mathrm{csm}}}{2 \pi l_{\mathrm{dS}}^{2}}\right|=\frac{\sqrt{l_{\mathrm{dS}}^{4}-4 \mu_{\mathrm{dS}} l_{\mathrm{dS}}^{2}}}{2 \pi l_{\mathrm{dS}}^{2} r_{\mathrm{dS}-\mathrm{bh}, \mathrm{csm}}}
$$

for the SdS spacetime. In [11, 12], the mass $M$ of the black hole has been evaluated by using the surface counterterms method. One can regard the mass as the thermodynamical energy. Then

$$
E_{\mathrm{AdS}}=\frac{3 l_{\mathrm{AdS}}^{2} V_{3}}{16 \kappa^{2}}\left(k^{2}+\frac{16 \mu_{\mathrm{AdS}}}{l_{\mathrm{AdS}}^{2}}\right)
$$

for the SAdS spacetime. Here $V_{3}$ is a volume of $3 \mathrm{~d}$ surface with unit radius. When the surface is $3 \mathrm{~d}$ sphere one has $k=2$ and $V_{3}=2 \pi^{2}$. On the other hand, for the SdS spacetime, one obtains

$$
E_{\mathrm{dS}}=\frac{3 \pi^{2} l_{\mathrm{dS}}^{2}}{2 \kappa^{2}}-\frac{6 \pi^{2} \mu_{\mathrm{dS}}}{\kappa^{2}}
$$


which corresponds to $k=2$ and $V_{3}=2 \pi^{2}$ case. The parts independent of $\mu_{\mathrm{AdS}, \mathrm{dS}}$ in (11) and (12) could be regarded as the Casimir energy of the bulk spacetime. Using the thermodynamical relation $d \mathcal{S}=\frac{d E}{T}$, we find the entropy

$$
\mathcal{S}_{\mathrm{AdS}}=\int \frac{d E}{T_{\mathrm{AdS}-\mathrm{bh}}}=\frac{4 V_{3} \pi r_{\mathrm{AdS}-\mathrm{bh}}^{3}}{\kappa^{2}}+\mathcal{S}_{0}^{\mathrm{AdS}}
$$

for the SAdS spacetime. Here $S_{0}^{\mathrm{AdS}}$ is a constant of the integration. If one requires that the entropy should vanish for the pure AdS with $\mu_{\text {AdS }}=$ $r_{\text {AdS-bh }}=0$, one arrives at $S_{0}^{\text {AdS }}=0$. It is funny that if the gravitational constant would be negative, then the entropy also would be negative.

On the other hand, when the spacetime is asymptotically dS, we have

$$
\mathcal{S}_{\mathrm{dS}}=\frac{4 V_{3} \pi r_{\mathrm{csm}}^{3}}{\kappa^{2}}+\mathcal{S}_{0}^{\mathrm{dS}}
$$

Here $S_{0}^{\mathrm{dS}}$ is a constant of the integration, again. When the spacetime is asymptotically de Sitter, the integration constant $\mathcal{S}_{0}^{\mathrm{dS}}$ may be determined by requiring that the entropy $\mathcal{S}_{\mathrm{dS}}$ should vanish in the Nariai limit $r_{\mathrm{dS}-\mathrm{bh}, \mathrm{csm}}^{2} \rightarrow$ $\frac{l_{\mathrm{dS}}^{2}}{2}$ :

$$
S_{0}^{\mathrm{dS}}=-\frac{2 V_{3} \pi l_{\mathrm{dS}}^{3}}{\sqrt{2} \kappa^{2}}
$$

Now, one can construct the same BHs in higher derivative gravity.

General action of $D=d+1$ dimensional $R^{2}$-gravity with cosmological constant is:

$$
S=\int d^{d+1} x \sqrt{-G}\left\{a R^{2}+b R_{\mu \nu} R^{\mu \nu}+c R_{\mu \nu \xi \sigma} R^{\mu \nu \xi \sigma}+\frac{1}{\kappa^{2}} R-\Lambda\right\} .
$$

When $c=0$ which is the case under consideration, Schwarzschild-(anti) de Sitter space is an exact solution:

$$
\begin{aligned}
d s^{2} & =G_{\mu \nu} d x^{\mu} d x^{\nu} \\
& =-\mathrm{e}^{2 \rho_{0}} d t^{2}+\mathrm{e}^{-2 \rho_{0}} d r^{2}+r^{2} \sum_{i, j}^{d-1} g_{i j} d x^{i} d x^{j}, \\
\mathrm{e}^{2 \rho} & =\frac{1}{r^{d-2}}\left(-\mu_{\mathrm{AdS}}+\frac{k r^{d-2}}{d-2}+\frac{r^{d}}{l_{\mathrm{AdS}}^{2}}\right) .
\end{aligned}
$$


The curvatures have the following form:

$$
R=-\frac{d(d+1)}{l_{\mathrm{AdS}}^{2}}, \quad R_{\mu \nu}=-\frac{d}{l_{\mathrm{AdS}}^{2}} G_{\mu \nu} .
$$

In (17), $\mu_{\mathrm{AdS}}$ is the parameter corresponding to mass and the length parameter $l_{\mathrm{AdS}}$ is found by solving the following equation:

$$
0=\frac{d^{2}(d+1)(d-3) a}{l_{\mathrm{AdS}}^{4}}+\frac{d^{2}(d-3) b}{l_{\mathrm{AdS}}^{4}}-\frac{d(d-1)}{\kappa^{2} l_{\mathrm{AdS}}^{2}}-\Lambda .
$$

We also assume $g_{i j}$ corresponds to the Einstein manifold, again. When $l_{\text {AdS }}^{2}$ is positive(negative), the spacetime is asymptotically anti-de Sitter (de Sitter). In the following, we concentrate on the case of $d=4$. Then Eq.([19) can be rewritten in the following form:

$$
\begin{aligned}
0 & =l_{\text {AdS }}^{4}+\frac{12 l_{\text {AdS }}^{2}}{\kappa^{2} \Lambda}-\frac{80 a+16 b}{\Lambda} \\
& =\left(l_{\text {AdS }}^{2}+\frac{5}{\kappa^{2} \Lambda}\right)^{2}-\frac{36}{\kappa^{4} \Lambda^{2}}-\frac{80 a+16 b}{\Lambda} .
\end{aligned}
$$

Therefore if and only if

$$
\frac{36}{\kappa^{4} \Lambda^{2}}+\frac{80 a+16 b}{\Lambda} \geq 0
$$

there are (real) solutions:

$$
\frac{1}{l_{\mathrm{AdS}}^{2}}=\frac{\frac{6}{\kappa^{2}} \pm \sqrt{\frac{36}{\kappa^{4}}+\Lambda(80 a+16 b)}}{80 a+16 b} .
$$

Eq.(20) tells that the signs of solution $l_{\text {AdS }}^{2}$ depend on the signs of $\kappa^{2} \Lambda$ and $\frac{80 a+16 b}{\Lambda}$.

1. When $80 a+16 b>0$ and $\Lambda>0$ or $80 a+16 b<0$ and $\Lambda<0$, one solution for $l_{\text {AdS }}^{2}(20)$ is positive but another is negative. Therefore there are two kinds of solution, the asymptotically AdS spacetime is first solution and another one is de Sitter space.

2. When $80 a+16 b>0$ and $\Lambda<0$, both of two solutions for $l_{\mathrm{AdS}}^{2}$ (20) are positive. Therefore these solutions correspond to anti-de Sitter space. 
3. When $80 a+16 b<0$ and $\Lambda>0$, both of two solutions for $l_{\mathrm{AdS}}^{2}=-l_{\mathrm{dS}}^{2}$ (20) are negative. Therefore these solutions correspond to de Sitter space.

Since there always appear two solutions, we now investigate the entropy. The entropy can be obtained by regarding the black hole mass as the thermodynamical mass. The thermodynamical mass is again obtained by using the surface counterterms method [8]. If the spacetime is asymptotically anti-de Sitter, the $(t, t)$ component of the surface energy momentum tensor has the following form for general $R^{2}$-gravity

$$
T^{t t}=\frac{3}{4 l_{\mathrm{AdS}} r^{6}}\left(\frac{1}{\kappa^{2}}-\frac{40 a}{l_{\mathrm{AdS}}^{2}}-\frac{8 b}{l_{\mathrm{AdS}}^{2}}-\frac{4 c}{l_{\mathrm{AdS}}^{2}}\right) .
$$

The black hole mass $M$ can be evaluated at the $3 \mathrm{~d}$ surface where $t$ is a constant:

$$
M=\int d x^{3} \sqrt{\tilde{g}} r^{3} \mathrm{e}^{\nu} T^{t t}\left(\xi_{t}\right)^{2} .
$$

Here $\zeta^{\mu}$ is a unit vector parallel with the time coordinate $t$ and therefore $\zeta^{t}=\mathrm{e}^{-\nu}\left(\zeta_{t}=\mathrm{e}^{\nu}\right)$ and $\zeta^{\mu}=\zeta_{\mu}=0(\mu \neq t)$. Note $\sqrt{\tilde{g}} \mathrm{e}^{\nu}=\sqrt{\operatorname{det} g_{m n}}$. Then for the asymptotically AdS spacetime

$$
M_{\mathrm{AdS}}=E_{\mathrm{AdS}}=\frac{3 l_{\mathrm{AdS}}^{2} V_{3}}{16}\left(\frac{1}{\kappa^{2}}-\frac{40 a}{l_{\mathrm{AdS}}^{2}}-\frac{8 b}{l_{\mathrm{AdS}}^{2}}-\frac{4 c}{l_{\mathrm{AdS}}^{2}}\right)\left(k^{2}+\frac{16 \mu_{\mathrm{AdS}}}{l_{\mathrm{AdS}}^{2}}\right)
$$

When $c=0$, in a way similar to the Einstein gravity case, we can obtain the expression for the entropy considering $M$ as a thermodynamical energy. When the spacetime is asymptotically anti-de Sitter, the entropy is given by

$$
\mathcal{S}_{\mathrm{AdS}}=\frac{V_{3} \pi r_{\mathrm{AdS}-\mathrm{bh}}^{3}}{2}\left(\frac{8}{\kappa^{2}}-\frac{320 a+64 b}{l_{\text {AdS }}^{2}}\right)+\mathcal{S}_{0}^{\mathrm{AdS}} .
$$

Here $S_{0}^{\text {AdS }}$ is a constant of the integration. If we assume that the entropy should vanish in the pure AdS, we have $S_{0}^{\text {AdS }}=0$. On the other hand, when the spacetime is asymptotically $\mathrm{dS}$, one has the following expressions for the thermodynamical energy

$$
M_{\mathrm{dS}}=E_{\mathrm{dS}}=\frac{3 l_{\mathrm{dS}}^{2} V_{3}}{16}\left(\frac{1}{\kappa^{2}}+\frac{40 a}{l_{\mathrm{dS}}^{2}}+\frac{8 b}{l_{\mathrm{dS}}^{2}}+\frac{4 c}{l_{\mathrm{dS}}^{2}}\right)\left(4-\frac{16 \mu_{\mathrm{dS}}}{l_{\mathrm{dS}}^{2}}\right)
$$


and the entropy for $c=0$ case,

$$
\mathcal{S}_{\mathrm{dS}}=\frac{V_{3} \pi r_{\mathrm{dS}-\mathrm{csm}}^{3}}{2}\left(\frac{8}{\kappa^{2}}+\frac{320 a+64 b}{l_{\mathrm{dS}}^{2}}\right)+\mathcal{S}_{0}^{\mathrm{dS}} .
$$

Here $l_{\mathrm{dS}}^{2}=-l_{\mathrm{AdS}}^{2}$. Since the horizon radius $r_{\mathrm{csm}}$ corresponds to the cosmological horizon, $r_{\mathrm{dS}-\mathrm{csm}}^{2}=l_{\mathrm{dS}}^{2}$ for pure de Sitter space. When the spacetime is asymptotically de Sitter, the integration constant $\mathcal{S}_{0}^{\mathrm{dS}}$ may be again determined by requiring that the entropy $\mathcal{S}_{\mathrm{dS}}$ should vanish in the Nariai limit:

$$
S_{0}^{\mathrm{dS}}=-\frac{V_{3} \pi l_{\mathrm{dS}}^{3}}{4 \sqrt{2}}\left(\frac{8}{\kappa^{2}}+\frac{320 a+64 b}{l_{\mathrm{dS}}^{2}}\right)
$$

Substituting the solution (22), we find

$$
\mathcal{S}_{\mathrm{AdS}, \mathrm{dS}}=\frac{V_{3} \pi r_{\mathrm{AdS}-\mathrm{bh}, \mathrm{dS}-\mathrm{csm}}^{3}}{2}\left(-\frac{16}{\kappa^{2}} \mp \sqrt{\frac{36}{\kappa^{4}}+\Lambda(80 a+16 b)}\right)+\mathcal{S}_{0}^{\mathrm{AdS}, \mathrm{d} \mathrm{S}} \text {. }
$$

Since the entropy should be positive, the lower sign in (22) or (30) should be chosen. Furthermore the condition that the entropy corresponding to the lower sign is really positive is

$$
\frac{20}{\kappa^{4} \Lambda^{2}}+\frac{80 a+16 b}{\Lambda} \geq 0
$$

In case that there are both of solutions which have positive entropy and negative one, the solution corresponding to negative entropy is unstable. The next interesting quantity is the free energy $F$. By using the thermodynamical relation $F=E-T \mathcal{S}$, one obtains the following expression for the asymptotically AdS spacetime case:

$$
\begin{aligned}
F_{\mathrm{AdS}}= & -V_{3}\left(\frac{1}{\kappa^{2}}-\frac{40 a+8 b}{l_{\mathrm{AdS}}^{2}}\right)\left(\frac{r_{\mathrm{AdS}-\mathrm{bh}}^{4}}{l_{\mathrm{AdS}}^{2}}-\frac{k}{2} r_{\mathrm{AdS}-\mathrm{bh}}^{2}-\frac{3 l_{\mathrm{AdS}}^{2} k^{2}}{16}\right) \\
& -\left(\frac{r_{\mathrm{AdS}-\mathrm{bh}}}{\pi l_{\mathrm{AdS}}^{2}}+\frac{k}{4 \pi r_{\mathrm{AdS}-\mathrm{bh}}}\right) \mathcal{S}_{0}^{\mathrm{AdS}}
\end{aligned}
$$

from (6), (9), (25) and (26). On the other hand, for the asymptotically dS spacetime case, using (8), (10), (27) and (28) one gets

$$
F_{\mathrm{dS}}=-V_{3}\left(\frac{1}{\kappa^{2}}+\frac{40 a+8 b}{l_{\mathrm{dS}}^{2}}\right)\left(\frac{r_{\mathrm{dS}-\mathrm{csm}}^{4}}{l_{\mathrm{dS}}^{2}}+r_{\mathrm{dS}-\mathrm{csm}}^{2}-\frac{3 l_{\mathrm{dS}}^{2}}{4}\right.
$$




$$
\left.-\frac{\sqrt{2} l_{\mathrm{dS}}}{r_{\mathrm{dS}-\mathrm{csm}}}\left(r_{\mathrm{dS}-\mathrm{csm}}^{2}-\frac{l_{\mathrm{dS}}^{2}}{2}\right)\right) .
$$

Then when the entropy vanishes, that is, $\frac{1}{\kappa^{2}}-\frac{40 a+8 b}{l_{\text {AdS }}^{2}}=0$ (for $S_{0}^{\text {AdS }}=0$ case) or $\frac{1}{\kappa^{2}}+\frac{40 a+8 b}{l_{\mathrm{dS}}^{2}}$, the free energy also vanishes. Then the negative entropy solution corresponds not to the minimum but to the maximum of the free energy. In the path integral formulation, the free energy corresponds to the product of the temperature and the action $S$ with the minus sign: $F=-T S$. Therefore the minus entropy corresponds to the minimum of the action. Therefore, this consideration indicates that the solution with negative entropy is instable.

Let us evaluate the action by substituting the classical solution (18) into the expression (16) with $d+1=5$. We first consider about the de Sitter case before AdS case. Then

$$
\begin{aligned}
S_{\mathrm{dS}} & =V_{3} \int_{0}^{\beta} d t \int_{r_{\mathrm{dS}-\mathrm{bh}}}^{r_{\mathrm{dS}-\mathrm{csm}}} d r r^{3}\left(\frac{400 a+80 b}{l_{\mathrm{dS}}^{4}}+\frac{20}{\kappa^{2} l_{\mathrm{dS}}^{2}}-\Lambda\right) \\
& =2 \beta V_{3}\left(r_{\mathrm{dS}-\mathrm{csm}}^{4}-r_{\mathrm{dS}-\mathrm{bh}}^{4}\right)\left(\frac{40 a+8 b}{l_{\mathrm{dS}}^{4}}+\frac{1}{\kappa^{2} l_{\mathrm{dS}}^{2}}\right) \\
& =2 \beta V_{3}\left(2 r_{\mathrm{dS}-\mathrm{csm}}^{2}-l_{\mathrm{dS}}^{2}\right)\left(\frac{1}{\kappa^{2}}+\frac{40 a+8 b}{l_{\mathrm{dS}}^{2}}\right) .
\end{aligned}
$$

Here $\beta$ is the period of the time coordinate. In the second line, Eq.(19) with $d=4$ and $l_{\mathrm{AdS}}^{2}=-l_{\mathrm{dS}}^{2}$ is used. In the last line, Eqs.(17) and (8) are used. The action (34) surely vanishes when the entropy vanishes $\left(\left(\frac{1}{\kappa^{2}}+\frac{40 a+8 b}{l_{\mathrm{dS}}^{2}}\right)=0\right)$. Since $2 r_{\mathrm{dS}-\mathrm{csm}}^{2}-l_{\mathrm{dS}}^{2}>0$, the action is positive (negative) when the entropy is positive (negative) (the sign in front of the action has been chosen so that the partition function $Z$ is given by $Z \sim \mathrm{e}^{S}$ ). Therefore the solution with negative entropy should be really instable.

In case that the spacetime is asymptotically anti-de Sitter, the action itself diverges due to the infinite volume of the AdS. In order to regularize the action, we cut off the spacetime at $r=r_{\max }$ and put the surface counterterms there[9]:

$$
\begin{aligned}
S_{b} & =S_{b}^{(1)}+S_{b}^{(2)} \\
S_{b}^{(1)} & =\int d^{4} x \sqrt{-g}\left[4 a \nabla_{\mu} n^{\mu} A+2 b\left(n_{\mu} n_{\nu} \nabla_{\sigma} n^{\sigma}+\nabla_{\mu} n_{\nu}\right) B^{\mu \nu}\right.
\end{aligned}
$$




$$
\begin{aligned}
& \left.+8 c n_{\mu} n_{\nu} \nabla_{\tau} n_{\sigma} C^{\mu \tau \nu \sigma}+\frac{2}{\kappa^{2}} \nabla_{\mu} n^{\mu}\right] \\
S_{b}^{(2)}= & -\int d^{4} x \sqrt{-g_{(4)}}\left(\eta_{1}+\eta_{2} R_{(4)}\right) .
\end{aligned}
$$

Here $g_{(4)}$ is the metric induced on the boundary and $R_{(4)}$ is the scalar curvature given by $g_{(4)} \cdot n^{\mu}$ is the normal outward unit vector to the boundary surface. $\eta_{1}$ and $\eta_{2}$ are constants given by

$$
\eta_{1}=\frac{6}{l_{\mathrm{AdS}}}\left(\frac{1}{\kappa^{2}}-\frac{40 a+8 b}{l_{\mathrm{AdS}}^{2}}\right), \quad \eta_{2}=k l_{\mathrm{AdS}}\left(\frac{1}{\kappa^{2}}-\frac{40 a+8 b}{l_{\mathrm{AdS}}^{2}}\right) .
$$

Then the actions have the following forms:

$$
\begin{aligned}
S_{\mathrm{AdS}}= & -2 \beta V_{3}\left(r_{\mathrm{max}}^{4}-r_{\mathrm{AdS}-\mathrm{bh}}^{4}\right)\left(\frac{1}{\kappa^{2} l_{\mathrm{AdS}}^{2}}-\frac{40 a+8 b}{l_{\mathrm{AdS}}^{4}}\right) \\
S_{b}= & \beta V_{3} \mathrm{e}^{\nu\left(r_{\max }\right)}\left(\frac{1}{\kappa^{2} l_{\mathrm{AdS}}^{2}}-\frac{40 a+8 b}{l_{\mathrm{AdS}}^{4}}\right) r_{\max }^{3} \\
& \times\left(-2 \nabla_{\mu} n^{\mu}+\frac{6}{l_{\mathrm{AdS}}}+k l_{\mathrm{AdS}} R_{(4)}\right) .
\end{aligned}
$$

Then total action including the surface counterterms vanishes again when the entropy vanishes $\left(\left(\frac{1}{\kappa^{2}}-\frac{40 a+8 b}{l_{\text {AdS }}^{2}}\right)=0\right)$.

The obtained results are summarized in Figure 1: The parameters region outside the curve $\Lambda(80 a+16 b)+\frac{36}{\kappa^{4}}=0$ is presumably forbidden since $l_{\text {AdS }}^{2}$ becomes complex number there. (Nevertheless, in principle there is possibility of realization of real well-defined four-dimensional brane even in the situation when $5 \mathrm{~d}$ bulk space has complex curvature). In the region between two curves, $\Lambda(80 a+16 b)+\frac{20}{\kappa^{4}}=0$ and $\Lambda(80 a+16 b)+\frac{36}{\kappa^{4}}=0$, the entropy is always negative. As it follows from the action principle these regions are not realistic as corresponding solutions are not stable. Asymptotically anti-de Sitter (de Sitter) spacetime with $l_{\text {AdS }}^{2}>0\left(l_{\text {AdS }}^{2}<0\right)$ can appear as a solution of Eq.(20) even if $\Lambda>0(\Lambda<0)$. These solutions have always negative entropy and again are not stable as it was shown above. As a result, as in the Einstein theory, the anti-de Sitter (de Sitter) spacetime corresponds to the negative (positive) $\Lambda$. 


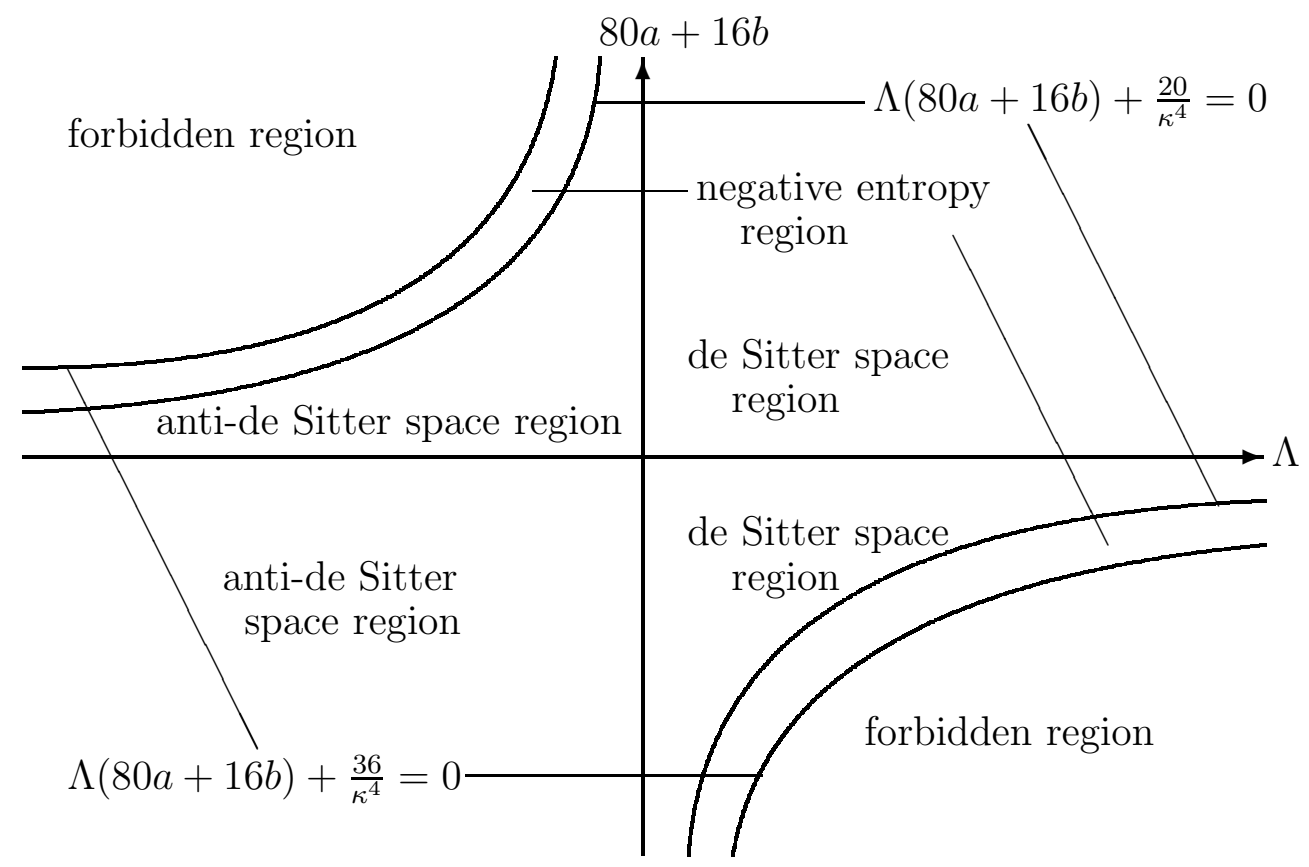

Figure 1: The relation between the parameters and the spacetime structure 


\section{The brane motion and dual CFTs}

Having in mind AdS/CFT and dS/CFT correspondence, one can investigate the structure in Fig.1 from the dual CFT side. For this purpose, we consider the motion of the brane in the Schwarzschild-AdS or dS black hole spacetime. We call that the brane is time (space)-like if the vector normal to the brane is space (time)-like. We now assume the brane exists at $r=\tilde{a}(\tau)$, where $\tau$ is the proper time on the brane. Then the Hubble "constant" $H$ is defined by

$$
H=\frac{1}{\tilde{a}} \frac{d \tilde{a}}{d \tau},
$$

the motion of the time-like brane obeys the following equation

$$
H^{2}=\frac{1}{l_{\mathrm{dS}, \mathrm{AdS}}^{2}}-\frac{\mathrm{e}^{2 \rho(r=\tilde{a}(\tau))}}{\tilde{a}^{2}},
$$

and that of the space-like brane obeys [6]

$$
H^{2}=\frac{1}{l_{\mathrm{dS}, \mathrm{AdS}}^{2}}+\frac{\mathrm{e}^{2 \rho(r=\tilde{a}(\tau))}}{\tilde{a}^{2}} .
$$

Then in case of the SAdS background (2), one obtains

$$
\begin{aligned}
& H^{2}=-\frac{k}{2 \tilde{a}^{2}}+\frac{\mu_{\mathrm{AdS}}}{\tilde{a}^{4}} \text { (for the time-like brane) } \\
& \text { or } H^{2}=\frac{2}{l_{\mathrm{AdS}}^{2}}+\frac{k}{2 \tilde{a}^{2}}-\frac{\mu_{\mathrm{AdS}}}{\tilde{a}^{4}} \text { (for the space-like brane), }
\end{aligned}
$$

and in case of the SdS background (雨)

$$
\begin{aligned}
& H^{2}=\frac{2}{l_{\mathrm{dS}}^{2}}-\frac{1}{\tilde{a}^{2}}+\frac{\mu_{\mathrm{dS}}}{\tilde{a}^{4}} \text { (for the time-like brane) } \\
& \text { or } H^{2}=\frac{1}{\tilde{a}^{2}}-\frac{\mu_{\mathrm{dS}}}{\tilde{a}^{4}} \text { (for the space-like brane) }
\end{aligned}
$$

The above equations can be rewritten in the form of the FRW equation:

$$
\begin{aligned}
& H^{2}=-\frac{k}{2 \tilde{a}^{2}}+\frac{\kappa_{4}^{2}}{6} \frac{\tilde{E}_{\mathrm{AdS}}}{V} \text { (for the time-like brane) } \\
& \text { or } H^{2}=\frac{2}{l_{\mathrm{AdS}}^{2}}+\frac{k}{2 \tilde{a}^{2}}-\frac{\kappa_{4}^{2}}{6} \frac{\tilde{E}_{\mathrm{AdS}}}{V} \text { (for the space-like brane), } \\
& \tilde{E}_{\mathrm{AdS}}=\frac{6 \mu_{\mathrm{AdS}} V_{3}}{\kappa_{4}^{2} \tilde{a}}, \quad V=\tilde{a}^{3} V_{3}
\end{aligned}
$$


for the brane in SAdS and

$$
\begin{aligned}
& H^{2}=\frac{2}{l_{\mathrm{dS}}^{2}}-\frac{1}{\tilde{a}^{2}}+\frac{\kappa_{4}^{2}}{6} \frac{\tilde{E}_{\mathrm{dS}}}{V} \text { (for the time-like brane) } \\
& \text { or } H^{2}=\frac{1}{\tilde{a}^{2}}-\frac{\kappa_{4}^{2}}{6} \frac{\tilde{E}_{\mathrm{dS}}}{V} \text { (for the space-like brane) } \\
& \tilde{E}_{\mathrm{dS}}=\frac{6 \mu_{\mathrm{dS}} V_{3}}{\kappa_{4}^{2} \tilde{a}}
\end{aligned}
$$

for the brane in SdS. Here $V_{3}$ is the volume of the three-dimensional sphere with unit radius and $\kappa_{4}$ is the four-dimensional gravitational coupling. Usual choice for effective gravitational constant in higher derivative gravity is [9, 8, 13

$$
\begin{aligned}
& \frac{1}{\kappa_{4}^{2}}=\frac{1}{\tilde{\kappa}_{\mathrm{AdS}}^{2}} \equiv \frac{l_{\mathrm{AdS}}}{2}\left(\frac{1}{\kappa^{2}}-\frac{40 a}{l_{\mathrm{AdS}}^{2}}-\frac{8 b}{l_{\mathrm{AdS}}^{2}}\right) \\
& \text { or } \frac{1}{\kappa_{4}^{2}}=\frac{1}{\tilde{\kappa}_{\mathrm{dS}}^{2}} \equiv \frac{l_{\mathrm{dS}}}{2}\left(\frac{1}{\kappa^{2}}+\frac{40 a}{l_{\mathrm{dS}}^{2}}+\frac{8 b}{l_{\mathrm{dS}}^{2}}\right),
\end{aligned}
$$

but Eq. (46) can be a convention at this stage. The motion of the brane itself does not depend on the explicit definition of $\kappa_{4}^{2}$. Then even if the black hole entropy vanishes at $\frac{1}{\kappa^{2}}-\frac{40 a}{l_{\mathrm{AdS}}^{2}}-\frac{8 b}{l_{\mathrm{AdS}}^{2}}=0$ or $\frac{1}{\kappa^{2}}+\frac{40 a}{l_{\mathrm{dS}}^{2}}+\frac{8 b}{l_{\mathrm{dS}}^{2}}=0$, the motion of the brane is not singular.

By differentiating Eqs.(44) and (45), we obtain the second FRW equation:

$$
\dot{H}=\mp \frac{\kappa_{4}^{2}}{4}\left(\frac{\tilde{E}_{\mathrm{dS}, \mathrm{AdS}}}{V}+p_{\mathrm{dS}, \mathrm{AdS}}\right) \pm \frac{k}{2 \tilde{a}^{2}}, \quad p_{\mathrm{dS}, \mathrm{AdS}}=\frac{2 \mu_{\mathrm{dS}, \mathrm{AdS}}}{\tilde{a}^{4} \kappa_{4}^{2}}
$$

Here $k=2$ for the bulk dS and the upper sign corresponds to the time-like brane and the lower sign to the space-like brane. Since $3 p_{\mathrm{dS}, \mathrm{AdS}}=\tilde{E}_{\mathrm{dS}, \mathrm{AdS}} / V$, the trace of the energy-stress tensor arising from the matter on the brane vanishes, i.e., $T_{\mu}^{\text {matter } \mu_{\mu}}=0$. Thus, the matter on the brane can be regarded as radiation or, equivalently, as massless fields. In other words, dual field theory on the brane should be a conformal theory.

We now compare the brane energy $\tilde{E}_{\mathrm{AdS}}$ or $\tilde{E}_{\mathrm{dS}}(44)$ or 44 with the $5 \mathrm{~d}$ black hole mass (25) or (27). In case of the bulk AdS, if one subtracts the bulk Casimir energy

$$
E_{\mathrm{AdS} \mathrm{C}}=\frac{3 l_{\mathrm{AdS}}^{2} V_{3}}{16}\left(\frac{1}{\kappa^{2}}-\frac{40 a}{l_{\mathrm{AdS}}^{2}}-\frac{8 b}{l_{\mathrm{AdS}}^{2}}\right) k^{2}=\frac{3 l_{\mathrm{AdS}} V_{3}}{8} \frac{k^{2}}{\tilde{\kappa}_{\mathrm{AdS}}^{2}},
$$


from the black hole mass, one gets

$$
E_{\mathrm{AdS}}-E_{\mathrm{AdS} C}=\frac{l_{\mathrm{AdS}}}{\tilde{a}} \frac{\kappa_{4}^{2}}{\tilde{\kappa}_{\mathrm{AdS}}^{2}} \tilde{E}_{\mathrm{AdS}}
$$

Since $E_{\mathrm{AdS} C}$ is independent of the parameters characterizing the black hole, for example, of the radius of the black hole horizon, $E_{\mathrm{AdS} C}$ would be proper characteristic of the background where the black hole lies. Therefore one can regard that $E_{\text {AdS C }}$ should be a Casimir energy of AdS. Since the ratio of the time on the brane with the bulk time is given by $\frac{l_{\mathrm{AdS}}}{\tilde{a}}$ when the radius of the brane is large, it would be consistent the following choice $\kappa_{4}^{2}=\tilde{\kappa}_{\text {AdS }}^{2}$. Then when the black hole entropy vanishes at $\frac{1}{\kappa^{2}}-\frac{40 a}{l_{\mathrm{AdS}}^{2}}-\frac{8 b}{l_{\mathrm{AdS}}^{2}}=0$, the energy of the CFT on the brane also vanishes. And if the entropy is negative, the energy is also negative. In the following, we choose $\kappa_{4}^{2}=\widetilde{\kappa}_{\text {AdS }}^{2}$ for the case that the spacetime is asymptotically AdS.

On the other hand, in case of the bulk dS, even if we subtract the bulk Casimir energy

$$
E_{\mathrm{dSC}}=\frac{3 l_{\mathrm{dS}}^{2} V_{3}}{4}\left(\frac{1}{\kappa^{2}}+\frac{40 a}{l_{\mathrm{dS}}^{2}}+\frac{8 b}{l_{\mathrm{dS}}^{2}}\right)=\frac{3 l_{\mathrm{dS}} V_{3}}{2} \frac{1}{\tilde{\kappa}_{\mathrm{AdS}}^{2}},
$$

from the black hole mass, we find

$$
E_{\mathrm{dS}}-E_{\mathrm{dSC}}=-\frac{l_{\mathrm{dS}}}{\tilde{a}} \frac{\kappa_{4}^{2}}{\tilde{\kappa}_{\mathrm{dS}}^{2}} \tilde{E}_{\mathrm{dS}}
$$

Therefore there is a difference in sign. Then we should not identify the energy of the CFT on the brane with $\tilde{E}_{\mathrm{dS}}$ but with

$$
\hat{E}_{\mathrm{dS}} \equiv-\tilde{E}_{\mathrm{dS}} \text {. }
$$

For this choice, we may choose $\kappa_{4}^{2}=\tilde{\kappa}_{\mathrm{dS}}^{2}$. Then when the black hole entropy vanishes at $\frac{1}{\kappa^{2}}+\frac{40 a}{l_{\mathrm{dS}}^{2}}+\frac{8 b}{l_{\mathrm{dS}}^{2}}=0$, the energy of dual CFT on the brane also vanishes. When the entropy is positive (negative), the energy is also positive (negative). In the following, we choose $\kappa_{4}^{2}=\tilde{\kappa}_{\mathrm{dS}}^{2}$ for the bulk dS.

In case of the bulk AdS, one assumes that the total entropy $\mathcal{S}_{\text {AdS }}$ of the CFT on the brane is given by Eq. (26) with $\mathcal{S}_{0}^{\text {AdS }}=0$. Then if this entropy is constant during the cosmological evolution, the entropy density $s_{\text {AdS }}$ is given by

$$
s_{\mathrm{AdS}}=\frac{\mathcal{S}_{\mathrm{AdS}}}{\tilde{a}^{3} V_{3}}=\frac{8 \pi r_{\mathrm{AdS}-\mathrm{bh}}^{3}}{l_{\mathrm{AdS}} \tilde{\kappa}_{\mathrm{AdS}}^{2} \tilde{a}^{3}} .
$$


If we further assume that the brane temperature $T$ differs from the Hawking temperature $T_{\mathrm{AdS}-\mathrm{bh}}(9)$ (the expression does not change for the $R^{2}$-gravity with $c=0$ ) by the factor $l_{\text {AdS }} / \tilde{a}$, one finds

$$
T=\frac{l_{\mathrm{AdS}}}{\tilde{a}} T_{\mathrm{AdS}-\mathrm{bh}}=\frac{r_{\mathrm{AdS}-\mathrm{bh}}}{\pi \tilde{a} l_{\mathrm{AdS}}}+\frac{k l_{\mathrm{AdS}}}{4 \pi \tilde{a} r_{\mathrm{AdS}-\mathrm{bh}}}
$$

and, when $\tilde{a}=r_{\mathrm{AdS}-\mathrm{bh}}$, this implies that

$$
T=\frac{1}{\pi l_{\text {AdS }}}+\frac{k}{4 \pi r_{\text {AdS-bh }}^{2}} .
$$

If the energy and entropy are purely extensive, the quantity $\tilde{E}_{\mathrm{AdS}}+p_{\mathrm{AdS}} V-$ $T \mathcal{S}_{\text {AdS }}$ vanishes. In general, this condition does not hold and one can define the CFT Casimir energy $\tilde{E}_{\text {AdS C }}$ (we should distinguish it with the bulk Casimir energy (48)) as [14]

$$
\tilde{E}_{\mathrm{AdS} \mathrm{C}}=3\left(\tilde{E}_{\mathrm{AdS}}+p_{\mathrm{AdS}} V-T \mathcal{S}_{\mathrm{AdS}}\right) .
$$

Then

$$
\tilde{E}_{\mathrm{AdS} \mathrm{C}}=\frac{6 k r_{\mathrm{AdS}-\mathrm{bh}}^{2} V}{\kappa_{4}^{2} \tilde{a}^{4}}=\frac{6 k r_{\mathrm{AdS}-\mathrm{bh}}^{2} V_{3}}{\kappa_{4}^{2} \tilde{a}} .
$$

If the black hole entropy vanishes at $\frac{2}{l_{\mathrm{AdS}} \kappa_{4}^{2}}=\frac{2}{l_{\mathrm{AdS}} \tilde{\kappa}_{\mathrm{AdS}}^{2}}=\frac{1}{\kappa^{2}}-\frac{40 a}{l_{\mathrm{AdS}}^{2}}-\frac{8 b}{l_{\mathrm{AdS}}^{2}}=0$, the Casimir energy of the CFT on the brane also vanishes. Furthermore, when the black hole entropy is negative, the Casimir energy is negative (positive) if $k>0(k<0)$. When $k \neq 0$, we also find that

$$
\mathcal{S}_{\mathrm{AdS}}^{2}=\left(\frac{4 \pi \tilde{a}}{3 \sqrt{|2 k|}}\right)^{2} \tilde{E}_{\mathrm{AdS} \mathrm{C}}\left(2 \tilde{E}_{\mathrm{AdS}}-\tilde{E}_{\mathrm{AdSC}}\right) .
$$

This is the form of Cardy-Verlinde formula in AdS higher derivative gravity under consideration.

In case that the bulk is dS, the above arguments should be modified. First one needs to assume that the total entropy of the CFT on the brane is not given by Eq. (28) but by the black hole (not cosmological) horizon area

$$
\mathcal{S}_{\mathrm{dS}}^{p}=\frac{8 \pi r_{\mathrm{dS}-\mathrm{bh}}^{3} V_{3}}{l_{\mathrm{dS}} \tilde{\kappa}_{\mathrm{dS}}^{2}}
$$


We also assume that the temperature $T$ on the brane is given, in terms of the black hole (not cosmological) horizon radius, by

$$
T=\frac{l_{\mathrm{dS}}}{\tilde{a}} T_{\mathrm{dS}-\mathrm{bh}}=-\frac{r_{\mathrm{dS}-\mathrm{bh}}}{\pi \tilde{a} l_{\mathrm{dS}}}+\frac{l_{\mathrm{dS}}}{2 \pi \tilde{a} r_{\mathrm{dS}-\mathrm{bh}}} .
$$

Then the CFT Casimir energy $\tilde{E}_{\mathrm{dS} \text { C }}$ defined by

$$
\tilde{E}_{\mathrm{dSC}}=3\left(\tilde{E}_{\mathrm{dS}}+p_{\mathrm{dS}} V-T \mathcal{S}_{\mathrm{dS}}^{p}\right)
$$

has the following form

$$
\tilde{E}_{\mathrm{dSC}}=\frac{12 r_{\mathrm{dS}-\mathrm{bh}}^{2} V}{\kappa_{4}^{2} \tilde{a}^{4}} .
$$

If the black hole entropy vanishes at $\frac{2}{l_{\mathrm{dS}} \kappa_{4}^{2}}=\frac{2}{l_{\mathrm{dS}} \tilde{\kappa}_{\mathrm{dS}}^{2}}=\frac{1}{\kappa^{2}}+\frac{40 a}{l_{\mathrm{dS}}^{2}}+\frac{8 b}{l_{\mathrm{dS}}^{2}}=0$, the Casimir energy of the CFT on the brane also vanishes. One also arrives at Cardy-Verlinde formula in dS brane-world

$$
\left(\mathcal{S}_{\mathrm{dS}}^{p}\right)^{2}=\left(\frac{2 \pi \tilde{a}}{3}\right)^{2} \tilde{E}_{\mathrm{dS} \mathrm{C}}\left(2 \tilde{E}_{\mathrm{dS}}-\tilde{E}_{\mathrm{dS} \mathrm{C}}\right)
$$

The above formula (63) seems to be identical with the corresponding expression (58) for AdS bulk since $|k|=2$. Nevertheless, the meaning of the entropy and the energy seems to be different. In case of SAdS, these quantities correspond to those of the whole spacetime of SAdS but in case of AdS they only correspond to the quantities proper for the black hole.

If, instead of (59) and (60), we define the entropy and the temperature in terms of the radius of the cosmological horizon:

$$
\mathcal{S}_{\mathrm{dS}}^{c}=\frac{8 \pi r_{\mathrm{dS}-\mathrm{csm}}^{3} V_{3}}{l_{\mathrm{dS}} \tilde{\kappa}_{\mathrm{dS}}^{2}}, \quad T^{c}=\frac{l_{\mathrm{dS}}}{\tilde{a}} T_{\mathrm{dS}-\mathrm{csm}}=\frac{r_{\mathrm{dS}-\mathrm{csm}}}{\pi \tilde{a} l_{\mathrm{dS}}}-\frac{l_{\mathrm{dS}}}{2 \pi \tilde{a} r_{\mathrm{dS}-\mathrm{csm}}},
$$

where $\mathcal{S}_{\mathrm{dS}}^{c}$ corresponds to Eq. (28) with $\mathcal{S}_{0}^{\mathrm{dS}}=0$, then by using $\hat{E}_{\mathrm{dS}}$ in (52) instead of $\tilde{E}_{\mathrm{dS}}$, the Casimir energy has the following form:

$$
\tilde{E}_{\mathrm{dSC}}^{c}=3\left(\hat{E}_{\mathrm{dS}}+p_{\mathrm{dS}} V-T^{c} \mathcal{S}_{\mathrm{dS}}^{c}\right)=-\frac{12 r_{\mathrm{dS}-\mathrm{bh}}^{2} V}{\kappa_{4}^{2} \tilde{a}^{4}} .
$$

Then, one gets

$$
\left(\mathcal{S}_{\mathrm{dS}}^{c}\right)^{2}=\left(\frac{2 \pi \tilde{a}}{3}\right)^{2} \tilde{E}_{\mathrm{dSC}}^{c}\left(2 \hat{E}_{\mathrm{dS}}+\tilde{E}_{\mathrm{dS} \mathrm{C}}^{c}\right)
$$


The sign in front of $\tilde{E}_{\mathrm{dS} \mathrm{C}}^{c}$ in the last factor is different from (63). However, the physical meaning of this relation is not clear. The conjecture could be that there are two types of dual CFT for SdS BH.

Thus, in this section it is considered the brane motion on (A)dS bulk and it is found the relation between energy, Casimir energy and entropy of dual CFT.

\section{Discussion}

In summary, the investigation of thermodynamics of five-dimensional (A)dS $\mathrm{BHs}$ in higher derivative gravity is presented. Entropy, free energy and mass (thermodynamical energy) for (A) $\mathrm{dS} \mathrm{BHs}$ is evaluated and their properties are discussed. It is shown that there exists the parameters region where the effective gravitational constant is getting negative (zero) and, as a result, the entropy becomes negative (zero) too. The arguments based on least action principle are given that negative entropy $\mathrm{BHs}$ are instable and their creation is highly suppressed. It is interesting to note that there exists RG interpretation for classical solutions of higher derivative gravity [15]. It could be that negative entropy BHs are not stable also from the point of view of holographic RG.

The motion of time (space)-like branes in SAdS or SdS BH background is considered. The corresponding equations of motion are presented in FRWform. The brane motion is not singular even when effective gravitational constant (BH entropy) is zero. It is demonstrated that dual QFT for higher derivative gravity model under consideration is CFT. Moreover, when BH entropy is zero the brane CFT energy is also zero. Finally, Cardy-Verlinde formula relating energy, entropy and Casimir energy of dual CFT is written in case of SAdS as well as SdS BHs.

Our study indicates that there are deep similarities between SAdS and SdS BHs and corresponding dual CFTs living on the branes. In particulary, Cardy-Verlinde formula looks the same in both cases, the BH entropy may be negative (zero) in both cases, etc. In this sense, our study provides further support to dS/CFT correspondence. As (A)dS BH thermodynamics is well-described here even in higher derivative gravity case, the natural next problem is the investigation of the properties of dual CFT. This will be discussed elsewhere. 


\section{Acknowledgements}

The authors would like to acknowledge helpful discussions with S.Ogushi. The work by SN is supported in part by the Ministry of Education, Science, Sports and Culture of Japan under the grant No. 13135208.

\section{References}

[1] J.M. Maldacena, Adv.Theor.Math.Phys. 2 (1998) 231; E. Witten, Adv.Theor.Math.Phys. 2 (1998) 253; S. Gubser, I. Klebanov and A. Polyakov, Phys.Lett. B428 (1998) 105, O. Aharony, S. Gubser, J. Maldacena, H. Ooguri and Y. Oz, Phys. Rept. 323 183, (2000), hepth/9905111.

[2] A. Strominger, hep-th/0106113; M. Spradlin, A. Strominger and A. Volovich, hep-th/0110007.

[3] C.M. Hull, JHEP 9807 (1998) 021, hep-th/9806146.

[4] E. Witten, hep-th/0106109.

[5] P.O. Mazur and E. Mottola, hep-th/0106151; M. Li, hep-th/0106184; S. Nojiri and S.D. Odintsov, Phys.Lett. B519 (2001) 145, hep-th/0106191; JHEP 0112 (2001) 033, hep-th/0107134; Phys.Lett. B523 (2001) 165, hep-th/0110064; E. Halyo, hep-th/0107169; C.M. Hull, hep-th/0109213; T. Shiromizu, D. Ida and T. Torii, hep-th/0109057; S. Cacciatori and D. Klemm, hep-th/0110031; B. McInnes, hep-th/0110062; Y.Gao, hepth/0107067; I.Y. Park, C.N. Pope and A. Sadrzadeh, hep-th/0110238; U. Danielsson, hep-th/0110265; Y. Myung, hep-th/0201176, hepth/0112140; R.-G. Cai, Y. Myung and Y. Zhang, hep-th/0110234; R.G. Cai, hep-th/0111093; A.C. Petkou and G. Siopsis, hep-th/0111085; A. Padilla, hep-th/0111247; A. Medved, hep-th/0111238; D. Youm, hep-th/0111276; B. Cunha, hep-th/0110169; M.Li and F. Lin, hepth/0111201; M. Dehghani, hep-th/0201128; R. Bousso, A. Maloney and A. Strominger, hep-th/0112218; M. Spradlin and A. Volovich, hep-

th/0112223; A. Ghezelbash, D. Ida, R. Mann and T. Shiromizu, hepth/0201004; E. Halyo, hep-th/0201174, hep-th/0203235. 
[6] S. Ogushi, Mod.Phys.Lett. A17 (2002) 51, hep-th/0111008.

[7] D. Kabat and G. Lifschytz, hep-th/0203083; G. Siopsis, hep-th/0203208; S.R. Das, hep-th/0202008; S. Ness and G. Siopsis, hep-th/0202096; S. Nojiri, S.D. Odintsov and S. Ogushi, hep-th/0202098; A. Ghezelbash and R.B. Mann, hep-th/0203003; A. Medved, hep-th/0202193; D. Klemm and L. Vanzo, hep-th/0203268; I. Brevik, K. Ghoroku, S.D. Odintsov and M. Yahiro, hep-th/0204066; S. Nojiri, S.D. Odintsov and A. Sugamoto, hep-th/0204065; H. Lee and Y. Myung, hep-th/0204083.

[8] M. Cvetič, S. Nojiri, and S. D. Odintsov, to appear in Nucl.Phys. B, hep-th/0112045; S. Nojiri and S.D. Odintsov, gr-qc/0112066, to appear in the Proceedings of the "1st Mexican Meeting on Mathematical and Experimental Physics", held at El Colegio Nacional, 10-14 September, 2001; J.E. Lidsey, S. Nojiri and S.D. Odintsov, hep-th/0202198.

[9] S. Nojiri, S.D. Odintsov and S. Ogushi, hep-th/0105117, Int.J.Mod. Phys. A16 (2001) 5085; hep-th/0108172, Phys.Rev. D65 (2001) 023521.

[10] I.L. Buchbinder, S.D. Odintsov and I.L. Shapiro, "Effective Action in Quantum Gravity", IOP Publishing, Bristol and Philadelphia, 1992.

[11] V. Balasubramanian and P. Kraus, Commun.Math.Phys. 208 (2000) 413.

[12] V. Balasubramanian, J. de Boer and D. Minic, hep-th/0110108.

[13] Y.M. Cho and I.P. Neupane, hep-th/0202140.

[14] E. Verlinde, hep-th/0008140.

[15] M. Fukuma and S. Matsuura, hep-th/0112037. 